\title{
Search with EGRET for a gamma ray line from the Galactic center
}

\author{
Anthony R. Pullen, Ranga-Ram Chary, and Marc Kamionkowski \\ California Institute of Technology, Mail Code 130-33, Pasadena, California 91125, USA \\ (Received 12 October 2006; published 17 September 2007)
}

\begin{abstract}
We search data from the Energetic Gamma Ray Experiment Telescope (EGRET) for a gamma-ray line in the energy range $0.1-10 \mathrm{GeV}$ from the $10^{\circ} \times 10^{\circ}$ region around the Galactic center. Our null results lead to upper limits to the line flux from the Galactic center. Such lines may have appeared if the dark matter in the Galactic halo is composed of weakly interacting massive particles (WIMPs) in the mass range $0.1-10 \mathrm{GeV}$. For a given dark-matter-halo model, our null search translates to upper limits to the WIMP two-photon annihilation cross section as a function of WIMP mass. We show that, for a toy model in which Majorana WIMPs in this mass range annihilate only to electron-positron pairs, these upper limits supersede those derived from measurements of the 511-keV line and continuum photons from internal bremsstrahlung at the Galactic center.
\end{abstract}

DOI: 10.1103/PhysRevD.76.063006

PACS numbers: 95.85.Pw, 95.35.+d, 98.35.Gi

\section{INTRODUCTION}

Weakly interacting massive particles (WIMPs) provide promising candidates for the dark matter in Galactic halos [1-3]. The most deeply explored WIMP candidate is the neutralino, the lightest superpartner in many supersymmetric extensions of the standard model [4]. Although the favored mass range for neutralinos is usually $\gtrsim 10 \mathrm{GeV}$, there are other WIMP candidates with masses in the 0.1$10 \mathrm{GeV}$ range. For example, neutralinos with masses as low as $6 \mathrm{GeV}$ are plausible if gaugino unification is not assumed [5]. Neutralinos with masses as low as $100 \mathrm{MeV}$ are plausible in the next-to-minimal supersymmetric standard model [6,7]. Also, scalar and spin- $1 / 2$ particles with masses in the $\mathrm{MeV}$ range have been considered [8] to explain the $511-\mathrm{keV}$ gamma-ray line observed by INTEGRAL $[9,10]$, a line whose strength, as explained in Ref. [8], has defied easy explanation from traditional astrophysics.

One way to detect WIMPs is to search for monoenergetic gamma rays produced by pair annihilation in the Galactic halo [11]. These gamma rays have energies equal to the WIMP mass $m_{\chi}$. Such a line spectrum could be easily distinguished from the continuum spectrum from more prosaic gamma-ray sources (e.g., cosmic-ray spallation), and thus serve as a "smoking gun" for dark-matter annihilation.

Since the dark-matter density is highest at the Galactic center, the flux of WIMP-annihilation photons should be greatest from that direction. On the other hand, the continuum background should also be highest from the Galactic center. We estimate that, for a Navarro-FrenkWhite profile [12], the WIMP-annihilation flux from the $10^{\circ} \times 10^{\circ}$ region from the Galactic center should exceed that from the Galactic anticenter by a factor $\sim 100$, while the flux of cosmic-ray-induced photons at energies $O(\mathrm{GeV})$ is only about 8 times higher from the Galactic center than from the Galactic anticenter. Thus, the Galactic center is the preferred place to look for a WIMP- annihilation signal. It is also the location of the $511-\mathrm{keV}$ anomaly that has motivated the consideration of lowermass WIMPs.

In this paper, we search data from the Energetic Gamma Ray Experiment Telescope (EGRET) [13] on the Compton Gamma Ray Observatory (CGRO) for a gamma-ray line in the energy range $100 \mathrm{MeV}$ to $10 \mathrm{GeV}$ from a $10^{\circ} \times 10^{\circ}$ region around the Galactic center. We found no evidence for a gamma-ray line from the Galactic center in this energy range. From these null results, we can bound the cross section $\langle\sigma v\rangle_{\gamma \gamma}$ for WIMP annihilation to two photons for WIMPs in this mass range.

The plan of our paper is as follows: In Sec. II, we discuss how EGRET data are cataloged. In Sec. III, we reconstruct from the EGRET data the differential flux of photons as a function of energy. In Sec. IV, we fit to the data a model of the flux produced by cosmic rays and point sources near the Galactic center. In Sec. V, we search for a line excess of photons from WIMP annihilation. In Sec. VI, we report upper limits to $\langle\sigma v\rangle_{\gamma \gamma}$ as a function of $m_{\chi}$ for WIMPs within the mass range of $0.1 \mathrm{GeV}$ to $10 \mathrm{GeV}$ for a variety of dark-matter-halo models. In Sec. VII, we show that in a toy model in which the WIMP annihilates only to electronpositron pairs, this upper limit is stronger over this mass range than limits derived from the 511-keV line and from lower-energy continuum gamma rays from internal bremsstrahlung.

\section{SOURCE OF DATA}

We obtained publicly available data from the CGRO Science Support Center (COSSC). ${ }^{1}$ We used the EGRET photon lists, which contain event lists of all photons detected during a given viewing period. The data that we used from these files are the photon's Galactic latitude, Galactic longitude, zenith angle, energy, and energy uncertainty. We also required the exposure files, which contain the detec-

\footnotetext{
${ }^{1}$ http://cossc.gsfc.nasa.gov/docs/cgro/cossc/egret/
} 
tor's effective area multiplied by the viewing time of the detector for a particular viewing period multiplied by EGRET's 1-sr field of view. The exposure is provided as a function of latitude, longitude, and energy range. We also obtained the counts files, which contain the number of photons at various spatial coordinates and energy ranges within a viewing period. The energy bins, along with their respective energy ranges, are shown on the COSSC site.

\section{CONSTRUCTION OF GAMMA-RAY FLUX}

We begin by constructing the photon differential flux as a function of energy. We use data only from a square region on the sky from $-5^{\circ}$ to $5^{\circ}$ Galactic longitude and $-5^{\circ}$ to $5^{\circ}$ Galactic latitude. Each viewing period covers a particular region of the sky, and there were 34 viewing periods for our region of interest. These viewing periods were found using Table I in the third EGRET catalog [14] and are listed in Table I.

The differential photon flux can be determined from the counts files provided by EGRET, but these provide only counts in 10 energy bins, each with a width comparable to the photon energy in that bin. However, we will below search for lines with energies spanning the full energy range. This analysis is performed (as discussed below) by fitting the measured photon distribution to a continuum plus a line broadened by a Gaussian, consistent with the instrumental resolution, about each central line energy. We therefore work with the EGRET events and exposure files, which list an energy and effective exposure, respectively, for each photon, and reconstruct the differential energy flux in 119 energy bins. Before doing so, however, we first construct the differential energy flux from the events files with the same 10 bins as in the EGRET counts files, to be sure that our event-file analysis recovers the EGRET counts files, the most commonly used EGRET data product.

We first split the data into the 10 energy bins used by EGRET. Since the exposure files record a photon index value of 2.1 for the photon distribution (a value more or less consistent with the fluxes arrived at in Figs. 1 and 2), the average energy $E_{\text {avg }}$ of photons in an energy bin $\left[E_{\min }, E_{\max }\right]$ is

$$
E_{\text {avg }}=11 \times \frac{E_{\min }^{-0.1}-E_{\max }^{-0.1}}{E_{\min }^{-1.1}-E_{\max }^{-1.1}} \mathrm{MeV} .
$$

TABLE I. Viewing periods used in analysis. The more dominant viewing periods are in bold and have an exposure of $>10^{6} \mathrm{~cm}^{2} \mathrm{~s}$ sr at $150-300 \mathrm{MeV}$, over our region of interest.

\begin{tabular}{rrrrrrr}
\hline \hline $\mathbf{5 . 0}$ & $\mathbf{7 . 2}$ & $\mathbf{1 3 . 1}$ & $\mathbf{1 6 . 0}$ & 20.0 & 23.0 & $\mathbf{2 7 . 0}$ \\
35.0 & 38.0 & 42.0 & 43.0 & $\mathbf{2 0 9 . 0}$ & $\mathbf{2 1 0 . 0}$ & $\mathbf{2 1 4 . 0}$ \\
219.0 & $\mathbf{2 2 3 . 0}$ & $\mathbf{2 2 6 . 0}$ & 229.0 & $\mathbf{2 2 9 . 5}$ & 231.0 & $\mathbf{2 3 2 . 0}$ \\
$\mathbf{3 0 2 . 3}$ & $\mathbf{3 2 3 . 0}$ & $\mathbf{3 2 4 . 0}$ & $\mathbf{3 3 0 . 0}$ & $\mathbf{3 3 2 . 0}$ & $\mathbf{3 3 4 . 0}$ & $\mathbf{3 3 6 . 5}$ \\
339.0 & $\mathbf{4 2 1 . 0}$ & $\mathbf{4 2 2 . 0}$ & $\mathbf{4 2 3 . 0}$ & $\mathbf{4 2 3 . 5}$ & $\mathbf{4 2 9 . 0}$ & \\
\hline \hline
\end{tabular}

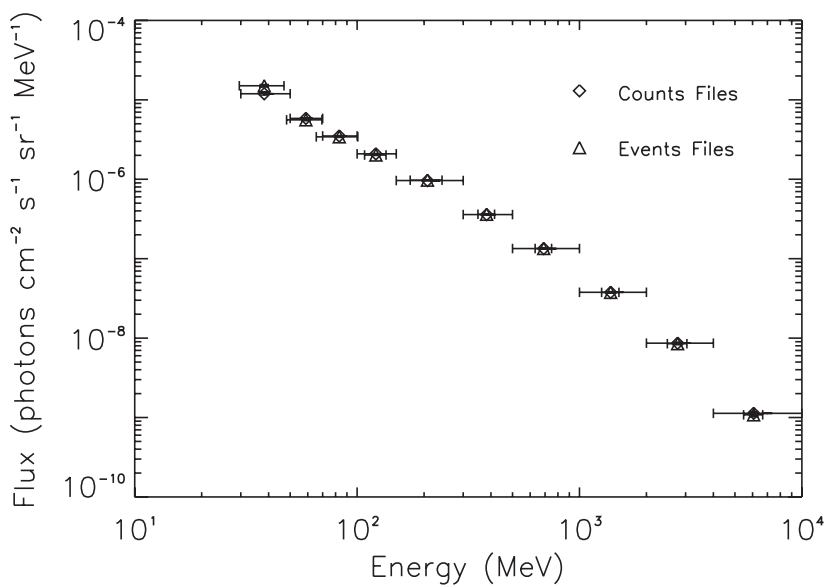

FIG. 1. The differential flux within ten energy bins with error bars denoting energy uncertainty for events data and half-bin sizes for counts data.

Variation of the photon index values over the range $[1.7,2.7]$ only changes $E_{\text {avg }}$ by $\sim 1 \%$ for these energy bins and by $\sim 0.01 \%$ for the 119 smaller energy bins. This variation also only changes the average exposures by less than $10 \%$, which does not affect our final results significantly. Thus, our assumption of a value of 2.1 for the photon index is a reasonable one.

We calculate the differential flux (photons $\mathrm{cm}^{-2} \mathrm{~s}^{-1} \mathrm{sr}^{-1} \mathrm{MeV}^{-1}$ ) from the counts files using

$$
F\left(E_{i}\right)=\frac{n\left(E_{i}\right)}{\varepsilon\left(E_{i}\right) \Delta E_{i}}
$$

where $E_{i}$ is the average energy of one of the ten large energy bins, $n\left(E_{i}\right)$ is the number of photons within that energy bin, $\varepsilon\left(E_{i}\right)$ is the total exposure from the exposure files over the viewing region within that energy bin, and $\Delta E_{i}$ is the size of the energy bin. The quantities $n\left(E_{i}\right)$ and $\varepsilon\left(E_{i}\right)$ are both summed over all viewing periods and all

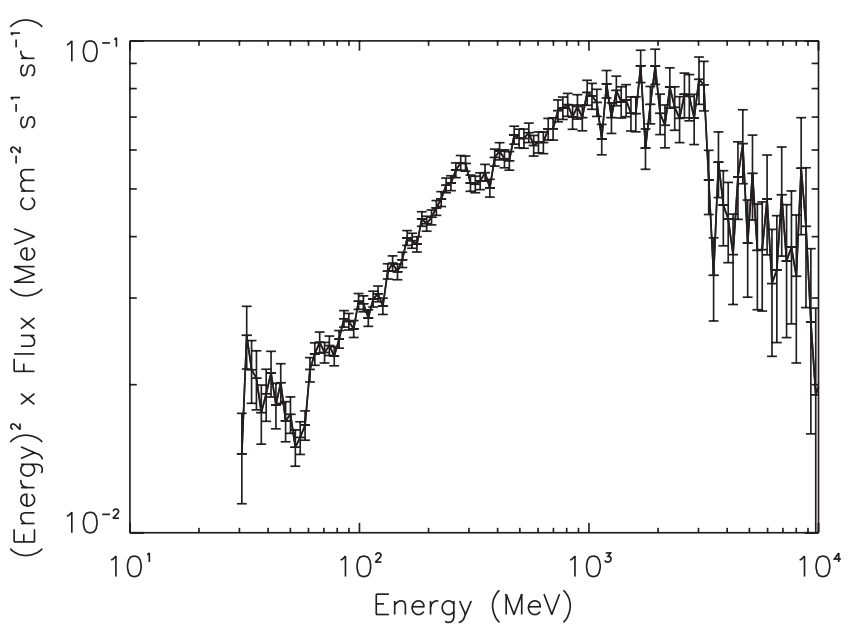

FIG. 2. The photon differential flux using 120 energy bins. 
positions within the region of interest. The uncertainty $\sigma_{F}\left(E_{i}\right)$ in the flux is

$$
\sigma_{F}\left(E_{i}\right)=\frac{\sqrt{n\left(E_{i}\right)}}{\varepsilon\left(E_{i}\right) \Delta E_{i}}
$$

We assume Gaussian errors in the photon energy. The energy uncertainty is just the median of the energy uncertainties of the individual photons within that energy bin, taken from the events data.

We then constructed from the events file the photon number $n\left(E_{i}\right)$ in each counts-file energy bin. We found that, in order to reproduce the counts data from the events file, we needed to reject photons with zenith angles greater than $100^{\circ}$ and energy uncertainties greater than $40 \%$ of the photon energy. This zenith cut also rejects albedo gamma rays from the Earth's atmosphere. The photon differential fluxes obtained from both the counts files and the events files (binned in the same way as the counts files) are shown in Fig. 1. We were not able to match the counts- and eventsfile photon numbers at the first energy bin to within $25 \%$. However, for reasons discussed below, we discarded this energy bin (below $0.1 \mathrm{GeV}$ ) from our analysis.

We then proceeded to construct the differential flux from the events files, applying the same photon cuts, with narrower bins, to facilitate the analysis in Sec. V. We split the data into 119 energy bins, with each bin ranging in energy from $E_{\min , i}=30 \times 1.05^{i} \mathrm{MeV}$ to $E_{\mathrm{max}, i}=30 \times$ $1.05^{i+1} \mathrm{MeV}$, where $i$ ranges from 0 to 118 . To calculate exposures, we interpolated $\log \left[\varepsilon\left(E_{n}\right)\right]$ over $\log \left(E_{n}\right)$, where $E_{n}$ is an average energy for a large energy bin $n$, and $\varepsilon\left(E_{n}\right)$ is the same exposure for the large bin $n$ used for the ten large bins earlier. Figure 14 of Ref. [13] shows that the exposures do not vary rapidly for energies $\gtrsim 0.1 \mathrm{GeV}$, and so this interpolation should be sufficient for our purposes. The flux is shown in Fig. 2. We note that Figs. 1 and 2 agree with EGRET's measurement of the diffuse gamma-ray spectrum in the same region of sky, shown in Fig. 4 of Ref. [15]. We also note a bump in the differential flux in Fig. 2 at around $3 \mathrm{GeV}$. We believe this artifact is due to the miscalibration of Class B photon events [16].

\section{DETERMINATION OF CONTINUUM GAMMA-RAY FLUX}

The line we seek is an excess over a continuum, and we must therefore model that continuum before we can search for an excess. Our aim in this section is thus to find a simple functional form that accurately models the continuum over the resolution scales of the instrument. A simple linear interpolation over each space of several energy-resolution elements would be sufficient, but we instead consider several astrophysically motivated functional forms, although the details of the precise astrophysical origin for the continuum are not important for our search for a line excess.

We were able to find a good fit to the continuum by a linear combination of three astrophysical sources for the diffuse gamma-ray background from the Galaxy. In the first source, nuclear interactions, cosmic rays collide with nuclei in interstellar matter to produce neutral pions, which decay mostly into gamma rays [17]. The second process is bremsstrahlung from cosmic-ray electrons interacting with interstellar matter [17]. The third, interior-point-source emission, comes from unresolved point sources within our Galaxy, such as gamma-ray pulsars [18]. We also considered exterior-point-source emission [19] and inverse-Compton scattering of interstellar radiation from cosmic-ray electrons, but found that the first three sources listed above were sufficient to model the flux. Reference [17] gives the differential gamma-ray production functions for the nuclear and bremsstrahlung contributions. The production functions are for the cosmic-ray spectrum in the solar neighborhood. We assumed the production functions at the Galactic center are proportional to the production functions in the solar neighborhood.

The functional form of the differential flux to which we fitted the data was $F_{\text {fit }}(E)=\alpha F_{\text {nuc }}(E)+\beta F_{\text {brem }}(E)+$ $\sigma F_{\text {int }}(E)$, where $F_{\text {nuc }}(E), F_{\text {brem }}(E)$, and $F_{\text {int }}(E)$ are the differential photon fluxes from nuclear interactions, bremsstrahlung, and interior point sources, respectively, and $\alpha$, $\beta$, and $\sigma$ are amplitudes determined by fitting the data. The source functions for nuclear interactions and bremsstrahlung are

$$
\begin{gathered}
F_{\text {nuc }}(E)=\left\{\begin{array}{lc}
2.63\left(\frac{E}{\mathrm{GeV}}\right)^{-2.36} \exp \left[-0.45\left(\ln \left(\frac{E}{\mathrm{GeV}}\right)\right)^{2}\right] \mathrm{cm}^{-2} \mathrm{~s}^{-1} \mathrm{sr}^{-1} \mathrm{GeV}^{-1} & 0.01 \mathrm{GeV}<E<1.5 \mathrm{GeV}, \\
3.3\left(\frac{E}{\mathrm{GeV}}\right)^{-2.71} \mathrm{~cm}^{-2} \mathrm{~s}^{-1} \mathrm{sr}^{-1} \mathrm{GeV}^{-1} & 1.5 \mathrm{GeV}<E<7.0 \mathrm{GeV}, \\
4.6\left(\frac{E}{\mathrm{GeV}}\right)^{-2.86} \mathrm{~cm}^{-2} \mathrm{~s}^{-1} \mathrm{sr}^{-1} \mathrm{GeV}^{-1} & E>7.0 \mathrm{GeV}
\end{array}\right. \\
F_{\text {brem }}(E)=\left\{\begin{array}{lc}
0.44\left(\frac{E}{\mathrm{GeV}}\right)^{-2.35} \mathrm{~cm}^{-2} \mathrm{~s}^{-1} \mathrm{sr}^{-1} \mathrm{GeV}^{-1} & 0.01 \mathrm{GeV}<E<5.0 \mathrm{GeV} \\
2.1\left(\frac{E}{\mathrm{GeV}}\right)^{-3.3} \mathrm{~cm}^{-2} \mathrm{~s}^{-1} \mathrm{sr}^{-1} \mathrm{GeV}^{-1} & 5.0 \mathrm{GeV}<E<40 \mathrm{GeV}
\end{array}\right.
\end{gathered}
$$

We assume interior point sources to be gamma-ray pulsars. Three pulsars seen by EGRET were the Crab, Geminga, and Vela pulsars, which have photon indices of -2.12 , -1.42 , and -1.62 , respectively [18]. We approximate the photon index as having the average value of -1.7 , so that

$$
F_{\text {int }}(E)=\left(\frac{E}{\mathrm{GeV}}\right)^{-1.7} \mathrm{~cm}^{-2} \mathrm{~s}^{-1} \mathrm{sr}^{-1} \mathrm{GeV}^{-1} .
$$




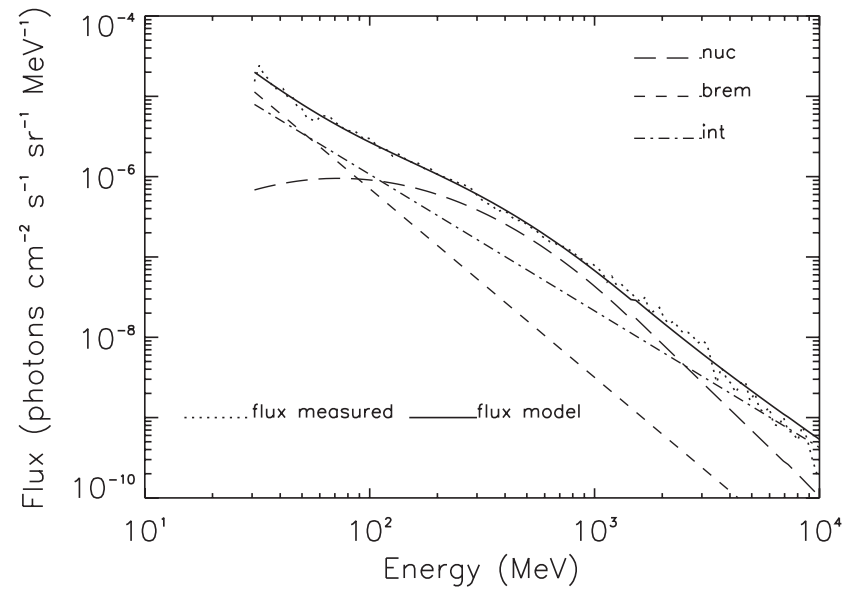

FIG. 3. The measured and model gamma-ray flux along with contributions from nuclear interactions (nuc), bremsstrahlung (brem), and interior point sources (int).

The fitted flux $\left[F_{\text {fit }}\left(E_{i}\right)\right]$ and the subsequent contributions from each physical process are shown in Fig. 3.

\section{ANALYSIS OF EXCESS PHOTONS IN GAMMA- RAY SPECTRUM}

We next construct a residual number of counts by subtracting the fitted number $N_{\text {fit }}\left(E_{i}\right)=F_{\text {fit }}\left(E_{i}\right) \varepsilon\left(E_{i}\right) \Delta E_{i}$ from the observed number $N\left(E_{i}\right)$ of counts. The counts $N\left(E_{i}\right)$ and $N_{\text {fit }}\left(E_{i}\right)$ are displayed in Fig. 4.

We take the residual spectrum to be the upper limit to the number of photons in each energy bin that could come from WIMP annihilation. However, to search for the signal we must take into account the finite energy resolution. With infinite energy resolution, the WIMP-annihilation excess would appear as a monochromatic peak over a smooth background distribution. However, because of energy uncertainties, each photon captured by EGRET will

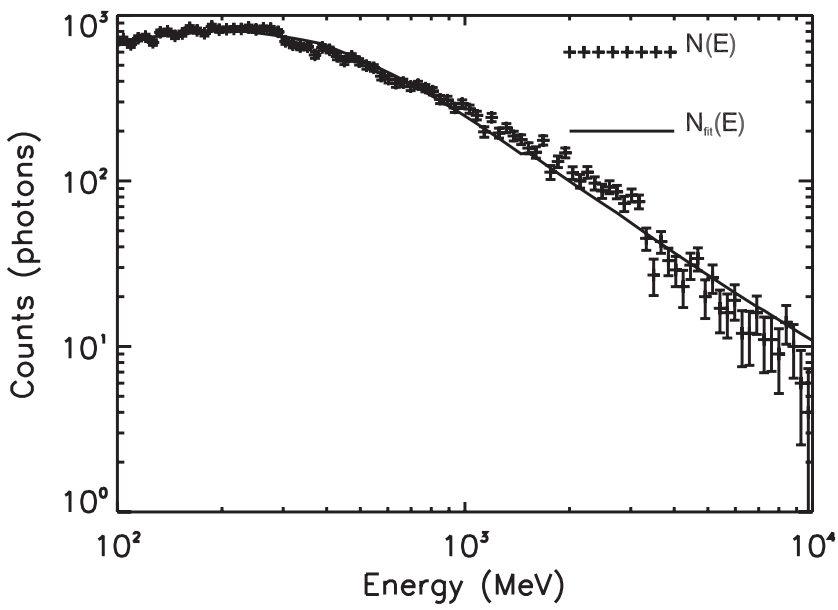

FIG. 4. The spectrum of actual counts, $N\left(E_{i}\right)$, and the fitted spectrum, $N_{\text {fit }}\left(E_{i}\right)$. appear to have an energy equal to its true energy plus an error, which we take to be Gaussian. Thus, monochromatic photons will be spread over neighboring energy bins. Because our bins are logarithmically spaced, the Gaussian will appear skewed, but it will still be distinguishable from the background spectrum.

Suppose our true spectrum before measurement consists of a continuum $C\left(E_{i}\right)$ produced by background radiation and an excess $N_{p}$ of photons with energy $E_{p}$. After measurement, the continuum will change shape but remain smooth, while the excess will spread out as a Gaussian profile over multiple bins. The Gaussian skews negligibly, so we approximate the excess as a standard Gaussian. Thus, we model the data $D\left(E_{i}\right)$ as

$$
D\left(E_{i}\right)=C\left(E_{i}\right)+N_{p} f_{p}\left(E_{i}\right)
$$

where $f_{p}\left(E_{i}\right)$ is a normalized Gaussian of the form

$$
f_{p}\left(E_{i}\right)=\frac{\exp \left[-\left(E_{i}-E_{p}\right)^{2} / 2 \sigma_{E_{p}}^{2}\right]}{\sum_{l} \exp \left[-\left(E_{l}-E_{p}\right)^{2} / 2 \sigma_{E_{p}}^{2}\right]} .
$$

In Eq. (8), the denominator is summed over all energy bins within $3 \sigma_{E_{p}}$ of the Gaussian central energy $E_{p}$. The energy uncertainty $\sigma_{E_{p}}$ at energy $E_{p}$ is given by

$$
\sigma_{E_{p}}=\frac{E_{p}}{R\left(E_{p}\right)},
$$

where $R\left(E_{p}\right)$ is the dimensionless resolution at energy $E_{p}$. The fractional full width at half-maximum (percent FWHM), or $\sqrt{2} \ln 2$ times twice the reciprocal of the resolution, is shown for various energies in Fig. 20 in Ref. [13]. From the percent FWHM, we produce a table of resolution vs energy, shown in Table II. We calculate the resolution at each energy by interpolating $\log [R(E)]$ over $\log (E)$. Because the first value for $R$ given in Table II is for energy $E=100 \mathrm{MeV}$, we cannot extrapolate $\log (R)$ to lower energies with certainty. Therefore, we restrict our analysis to the energy interval $0.1 \mathrm{GeV}-10 \mathrm{GeV}$.

The number $N_{p}\left(E_{i}\right)$ can be deduced at each energy bin in the spectrum by solving Eq. (7) for $N_{p}$, assuming $D\left(E_{i}\right)$, $C\left(E_{i}\right)$, and $f_{p}\left(E_{i}\right)$ are known. Each $N_{p}\left(E_{i}\right)$ has an uncertainty,

TABLE II. Dimensionless resolution $R$ of EGRET at various energies.

\begin{tabular}{lr}
\hline \hline Energy $(\mathrm{MeV})$ & \multicolumn{1}{c}{$R$} \\
\hline 100 & 9.42 \\
200 & 11.21 \\
500 & 12.39 \\
1000 & 12.08 \\
3000 & 11.49 \\
10000 & 9.07 \\
\hline \hline
\end{tabular}




$$
\sigma_{N_{p}}\left(E_{i}\right)=\frac{\sqrt{C\left(E_{i}\right)}}{f_{p}\left(E_{i}\right)}
$$

due to continuum fluctuations. Most bins in the spectrum contain large numbers of photons. Therefore, we average $N_{p}$ using Gaussian statistics to calculate $\bar{N}_{p}$ and $\sigma_{\bar{N}_{p}}$, the value and uncertainty of the excess, for each energy bin $E_{p}$ greater than $100 \mathrm{MeV}$. The resulting ratio of $\bar{N}_{p}$ to $\sigma_{\bar{N}_{p}}$ is shown in Fig. 5.

Figure 5 does show statistically significant deviations of the data from our model for the continuum. To determine if this residual favors the Gaussian model, we compare $\chi^{2}$ for a Gaussian model to $\chi^{2}$ for a constant-excess model. We calculate $\chi^{2}$ for both models over a $\pm 3 \sigma_{E_{p}}$ range centered at the excess center. The Gaussian is $\bar{N}_{p} f_{p}$. We also compare the residual with a constant excess $N_{c}$, where $d N_{c} / d E$ is constant and $N_{c}$ is proportional to the energybin size. We normalize $N_{c}$ such that the lowest energy bin $3 \sigma_{E_{p}}$ from the Gaussian center has 10 photons. We compared $\chi^{2}$ for the excess at energies $E=210 \mathrm{MeV}$ and $E=$ $2000 \mathrm{MeV}$, two energies that have high excess photons to excess uncertainty ratios (see Fig. 5). At both energies we found $\chi^{2}$ to be smaller for the constant excess, a simpler model, than for the Gaussian. Thus, we show that the residual does not favor the Gaussian model, and we do not attribute any of these deviations to a WIMPannihilation line (see Fig. 6). Rather, it appears that there is some continuum contribution that our analysis has not taken into account.

We therefore use $\bar{N}_{p}$ to calculate an upper limit to the line flux. This line flux is different from the differential flux used in previous sections in that this flux is not divided by the energy-bin size. Since $\bar{N}_{p}$ has positive and negative values, we take the $2 \sigma$ upper limit to the line flux $\Phi_{u}\left(E_{p}\right)$ to be

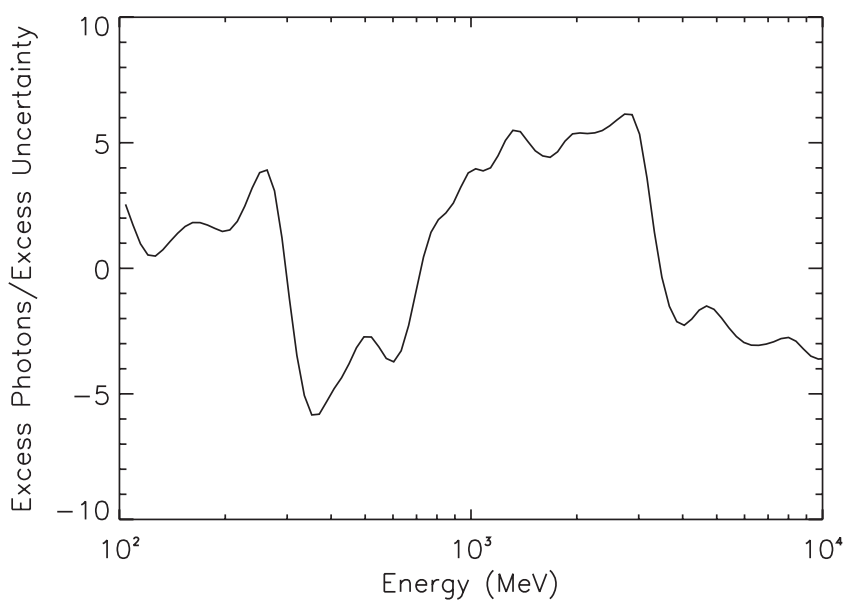

FIG. 5. Ratio of excess photons to the excess uncertainty.

$$
\Phi_{u}\left(E_{p}\right)= \begin{cases}\left(\bar{N}_{p}+2 \sigma_{\bar{N}_{p}}\right) / \varepsilon\left(E_{i}\right) & \bar{N}_{p} \geq 0, \\ 2 \sigma_{\bar{N}_{p}} / \varepsilon\left(E_{i}\right) & \bar{N}_{p}<0 .\end{cases}
$$

The $2 \sigma$ upper limit to the line flux is shown in Fig. 7.

We illustrate the reliability of the upper limit to the line flux by repeating the analysis in Sec. $\mathrm{V}$ for a slidingwindow continuum model. At each energy bin $E_{i}$ we fitted the diffuse flux data within 3 to $9 \sigma_{E_{i}}$ of $E_{i}$ to a single power law. The amplitude and index of the power law, which varied with energy bin, were then used to construct the background radiation continuum $C\left(E_{i}\right)$ in Sec. V needed to search for a line excess. No significant excess was found, and an upper limit to the line flux was determined. This $2 \sigma$ upper limit, shown in Fig. 7, agrees quite well with the previous upper limit in Sec. Vexcept around $3 \mathrm{GeV}$, where the previous upper bound is more conservative. To be
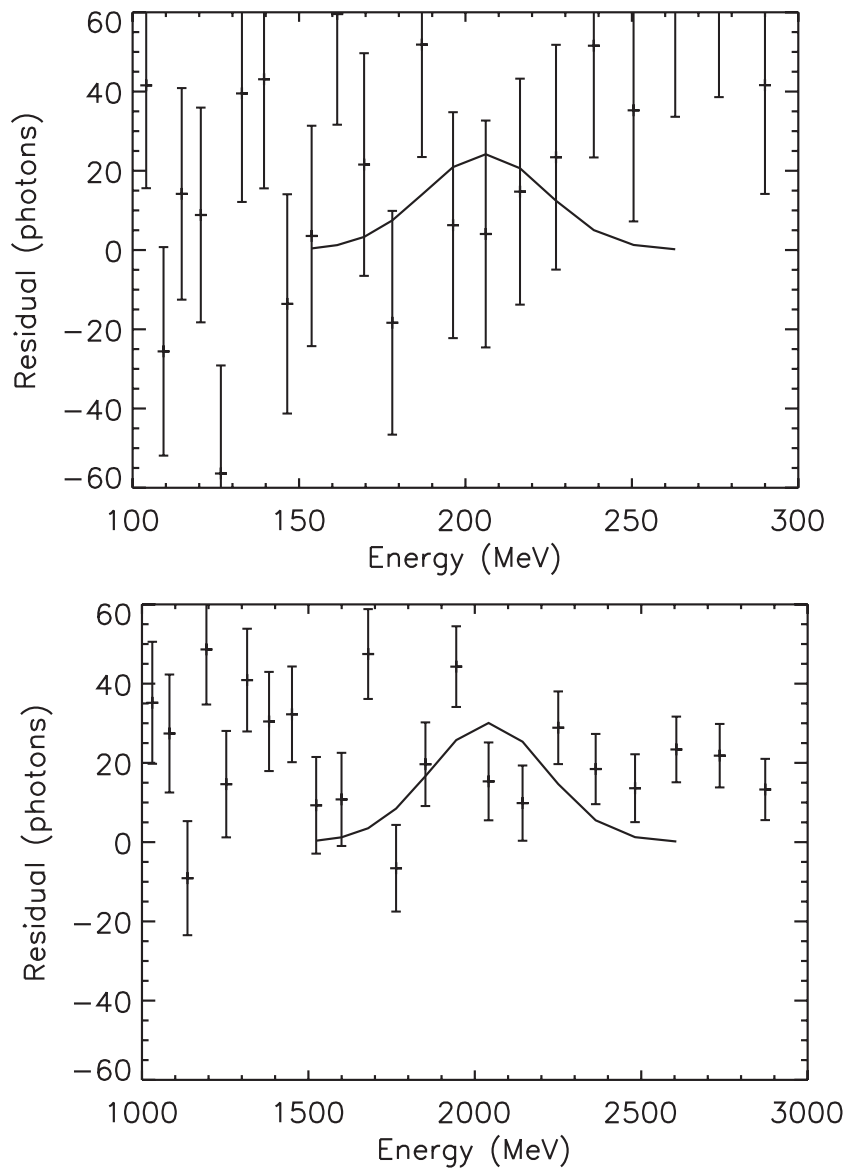

FIG. 6. The residual number of counts (crosses) and the expected Gaussian (solid curve) from a smeared line excess. The top panel shows the residual and Gaussian at $E=210 \mathrm{MeV}$, while the bottom panel shows the same at $E=2000 \mathrm{MeV}$. The ratio of excess photons to excess uncertainty is high at these energies. Notice in both panels the residual does not resemble the Gaussian. For $E=210 \mathrm{MeV}$ and $E=2000 \mathrm{MeV}$, respectively, $\chi^{2}$ for the Gaussian is 17.3 and 38.0 and $\chi^{2}$ for the constant excess is 11.0 and 23.9. Thus, the Gaussian model is not favored. 


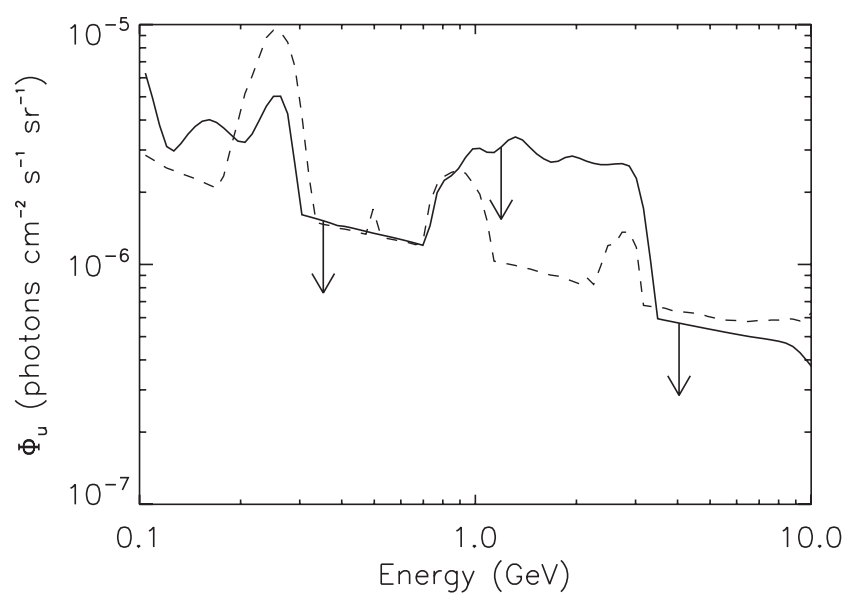

FIG. 7. Upper limits to the line flux $\Phi_{u}$ from the Galactic center. The solid line is the upper limit derived from the continuum model in Sec. IV. The dashed line is the upper limit derived from the sliding-window technique.

conservative, we chose the upper limit to the line flux from the multicomponent continuum fits, for the rest of our analysis.

\section{UPPER LIMITS TO THE ANNIHILATION CROSS SECTION}

If WIMPs comprise the Galactic halo, then the flux of line photons from WIMP annihilation is (for Majorana WIMPs)

$$
\Phi\left(E_{\gamma}=m_{\chi}\right)=\frac{\langle\sigma v\rangle_{\gamma \gamma}}{4 \pi m_{\chi}^{2}} \int_{\text {l.o.s }} \rho_{\chi}^{2} d l,
$$

where $\Phi$ is the line flux of photons in units of photons $\mathrm{cm}^{-2} \mathrm{~s}^{-1} \mathrm{sr}^{-1},\langle\sigma v\rangle_{\gamma \gamma}$ is the velocity-averaged cross section for the WIMP to annihilate to two photons, $m_{\chi}$ is the WIMP mass (which is equal to the photon energy $E_{\gamma}$ ), and $\rho_{\chi}$ is the density profile of the WIMP halo. The integral is along the line of sight, and $d l$ is the differential distance along the line of sight. The residual in the previous section gives the average line-of-sight line flux within a $10^{\circ} \times 10^{\circ}$ region around the Galactic center. Therefore, we integrate Eq. (12) over our viewing region to find the relation between $\langle\sigma v\rangle_{\gamma \gamma}$ and $m_{\chi}$.

The density profile of the WIMP halo must be known in order to integrate Eq. (12). The functional form of the halodensity profile is motivated by theory and simulations, with parameters chosen for consistency with the measured Milky Way rotation curve. We assume the following parametrization of the density profile:

$$
\rho(r)=\rho_{0} \frac{\left(r_{0} / a\right)^{\gamma}\left[1+\left(r_{0} / a\right)^{\alpha}\right]^{(\beta-\gamma) / \alpha}}{(r / a)^{\gamma}\left[1+(r / a)^{\alpha}\right]^{(\beta-\gamma) / \alpha}} .
$$

Here, $\rho_{0}$ is the local density of the halo at the Solar System; $r_{0}$ is the distance from the Solar System to the Galactic
TABLE III. Parameters for each profile type.

\begin{tabular}{lccccc}
\hline \hline Profile & $\alpha$ & $\beta$ & $\gamma$ & $\rho_{0}\left(\mathrm{GeV} / \mathrm{cm}^{3}\right)$ & $a(\mathrm{kpc})$ \\
\hline $\mathrm{Ka}$ & 2 & 3 & 0.2 & 0.4 & 11 \\
$\mathrm{~Kb}$ & 2 & 3 & 0.4 & 0.4 & 12 \\
$\mathrm{NFW}$ & 1 & 3 & 1 & 0.3 & 25 \\
Iso & 2 & 2 & 0 & 0.3 & 4 \\
\hline \hline
\end{tabular}

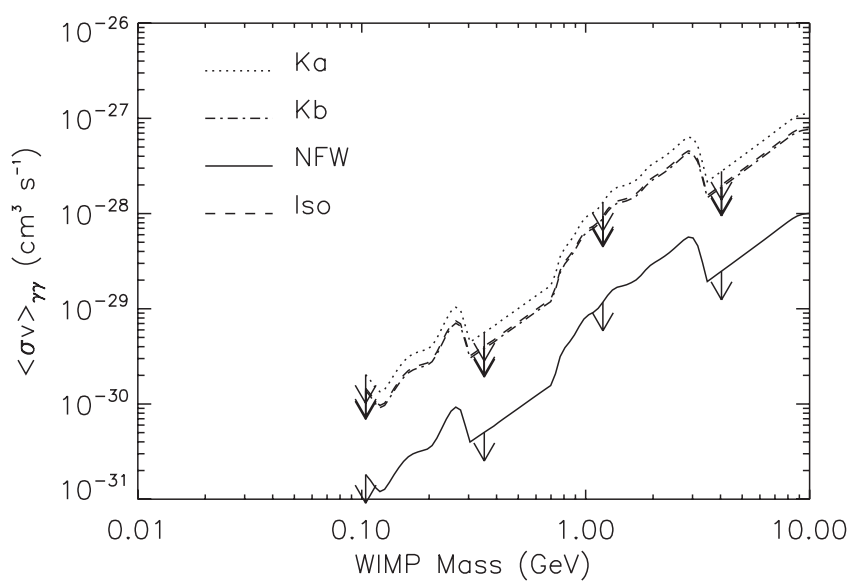

FIG. 8. The $2 \sigma$ upper limits to the velocity-averaged annihilation cross section $\langle\sigma v\rangle_{\gamma \gamma}$ as a function of WIMP mass for various halo-density profiles.

center, which we take to be $8.5 \mathrm{kpc} ; a$ is the core radius; and $\alpha, \beta$, and $\gamma$ are parameters that determine the halo model. Various combinations of $\alpha, \beta$, and $\gamma$ have been used in simulations and are of particular interest. We chose to study the $\mathrm{Ka}$ and $\mathrm{Kb}$ profiles proposed by Kravtsov et al. [20]; the NFW profile proposed by Navarro, Frenk, and White [12]; and the modified isothermal profile, or Iso, which is commonly used. These profiles are listed in Table III. The quantities $\rho_{0}$ and $a$ are chosen for each profile so that the profile will account for the Galactic rotation curve. These values are taken from Fig. 5 in Ref. [21]. We insert each of these profiles into Eq. (12) and integrate over our viewing region to find the line flux $\Phi$ in terms of $\langle\sigma v\rangle_{\gamma \gamma}$ and $m_{\chi}$.

The resulting upper limit to the annihilation cross section $\langle\sigma v\rangle_{\gamma \gamma}$ is shown in Fig. 8 as a function of WIMP mass $m_{\chi}$ for each halo model listed in Table III.

\section{DISCUSSION}

To illustrate the possible utility of this new bound, we consider a toy model in which WIMPs are Majorana fermions that couple to electrons via exchange of a scalar boson (the $U$ boson [22,23]) of mass $m_{U}$ (assumed to be much heavier than both WIMPs and electrons) through the Lagrangian density,

$$
\mathfrak{Q}=\frac{C_{U} f_{A e}}{2 m_{U}^{2}} \bar{\chi} \gamma_{\mu} \gamma_{5} \chi \bar{\psi}_{e} \gamma^{\mu} \gamma_{5} \psi_{e},
$$


where $C_{U}$ and $f_{A e}$ are axial couplings of the $U$ boson to the WIMP field $\chi$ and the electron field $\psi_{e}$, respectively. Annihilation of WIMPs with $O(\mathrm{MeV})$ masses to electron-positron pairs has been considered as a possible explanation [8] for the observed flux, $\Phi_{511}=9.9_{-2.1}^{+4.7} \times$ $10^{-4}$ photons $\mathrm{cm}^{-2} \mathrm{~s}^{-1}$ [9], of $511-\mathrm{keV}$ photons as measured at the Galactic center by the SPI camera on the INTEGRAL satellite. In this scenario, positrons from WIMP annihilation then annihilate with electrons in the interstellar medium to produce these $511-\mathrm{keV}$ photons. The annihilation rate-and therefore the cross section for annihilation to electron-positron pairs and thus the coupling $C_{U} f_{A e} / m_{U}^{2}$ - are determined by the flux of 511$\mathrm{keV}$ photons. More precisely, the $511-\mathrm{keV}$ flux determines an upper bound to this annihilation rate, cross section, and coupling, but we will here suppose the entire 511-keV flux to be from positrons from WIMP annihilation.

Reference [24] pointed out that, if WIMPs annihilate to electron-positron pairs, they can also undergo annihilation to an electron-positron-photon three-body final state, a process we refer to as internal bremsstrahlung. If $\langle\sigma v\rangle_{e^{+} e^{-}}$is the cross section for annihilation to electronpositron pairs (as calculated, e.g., in Refs. [22,23,25]), then the differential cross section for bremsstrahlung of a photon of energy $E_{\gamma}$ is

$$
\frac{d\langle\sigma v\rangle_{\mathrm{Br}}}{d E_{\gamma}}=\langle\sigma v\rangle_{e^{+} e^{-}} \frac{\alpha_{e}}{\pi} \frac{1}{E_{\gamma}}\left[\ln \left(\frac{s^{\prime}}{m_{e}^{2}}\right)-1\right]\left[1+\left(\frac{s^{\prime}}{s}\right)^{2}\right],
$$

where $s=4 m_{\chi}^{2}, s^{\prime}=4 m_{\chi}\left(m_{\chi}-E_{\gamma}\right)$, and $\alpha_{e}$ is the finestructure constant. The quantity $E_{\gamma}^{2} d\langle\sigma v\rangle_{\mathrm{Br}} / d E_{\gamma}$ increases roughly linearly with $E_{\gamma}$ for $E_{\gamma}<m_{\chi}$ and peaks at a value (for our WIMP mass range of $0.1-10 \mathrm{GeV}$ ) less than $10 \%$ smaller than the WIMP mass. The measured upper limits to the flux were approximated in Ref. [24] $E_{\gamma}^{2} d \Phi_{\mathrm{Br}} / d E_{\gamma} \lesssim$ $7 \times 10^{-3} \mathrm{MeV} \mathrm{cm}^{-2} \mathrm{~s}^{-1} \mathrm{sr}^{-1}$ over the energy range $1-$ $100 \mathrm{MeV}$. This flux was averaged over a region on the sky centered at the Galactic center from $-30^{\circ}$ to $30^{\circ}$ Galactic longitude and $-5^{\circ}$ to $5^{\circ}$ Galactic latitude. For the purposes of this illustrative exercise, we extend this bound up to $10 \mathrm{GeV}$ (roughly consistent with the line limit we have derived).

Each annihilation to an electron-positron pair produces two 511-keV photons either directly (7\% of all annihilations) or by producing positronium and decaying (23.3\% of all annihilations); the rest produce noncontributing continuum photons $[24,26]$. The resulting flux of $511-\mathrm{keV}$ photons is (for Majorana particles)

$$
\Phi_{511}=\frac{\xi\langle\sigma v\rangle_{e^{+} e^{-}}}{4 \pi m_{\chi}^{2}} \int \rho_{\chi}^{2} d l d \Omega,
$$

where $\xi=0.303$ is the fraction of positrons that undergo two-photon annihilation, the $d l$ integral is along the line of sight and the $d \Omega$ integral is over the SPI camera's field of view, a $16^{\circ}$-diameter circle around the Galactic center.
Likewise, the differential flux of photons from internal bremsstrahlung is

$$
\frac{d \Phi_{\mathrm{Br}}}{d E_{\gamma}}=\frac{d\langle\sigma v\rangle_{\mathrm{Br}} / d E_{\gamma}}{8 \pi m_{\chi}^{2} \Delta \Omega} \int \rho_{\chi}^{2} d l d \Omega,
$$

where $\Delta \Omega \simeq 0.182 \mathrm{sr}$ is the solid angle over the $60^{\circ}$ by $10^{\circ}$ Galactic region mentioned earlier.

The two-photon annihilation cross section $\langle\sigma v\rangle_{\gamma \gamma}$ for the Lagrangian of Eq. (14) is given by [27]

$$
\langle\sigma v\rangle_{\gamma \gamma}=\frac{\alpha_{e}^{2} m_{\chi}^{2} C_{U}^{2} f_{A e}^{2}}{\pi^{3} m_{U}^{4}}\left|I\left(\xi_{e}\right)\right|^{2},
$$

where $\xi_{e}=m_{e}^{2} / m_{\chi}^{2}, I\left(\xi_{e}\right)=\frac{1}{2}\left[1+\xi_{e} J\left(\xi_{e}\right)\right]$, and $J\left(\xi_{e}\right)$ is given by

$$
J\left(\xi_{e}\right)=\left(\frac{1}{2} \ln \frac{1+\sqrt{1-\xi_{e}}}{1-\sqrt{1-\xi_{e}}}-\frac{i \pi}{2}\right)^{2},
$$

for $\xi_{e} \leq 1$. For our WIMP mass range $0.1-10 \mathrm{GeV}, \xi_{e} \ll$ 1 and $I\left(\xi_{e}\right) \simeq 1 / 2$. The cross section for annihilation to electron-positron pairs $\langle\sigma v\rangle_{e^{+}} e^{-}$is given by $[23,25]$

$$
\langle\sigma v\rangle_{e^{+} e^{-}}=\frac{C_{U}^{2} f_{A e}^{2}}{2 \pi m_{U}^{4}}\left[\frac{4}{3} m_{\chi}^{2} \overline{v_{\chi}^{2}}+m_{e}^{2}\right]
$$

where $\overline{v_{\chi}^{2}}=\frac{3}{4} v_{c}^{2}$ is the mean-square center-of-mass velocity and $v_{c} \simeq 220 \mathrm{~km} / \mathrm{s}$ is the WIMP rotation speed, assuming the electron energy $E_{e}=m_{\chi} \gg m_{e}$ and $m_{U} \gg m_{\chi}$. We use Eqs. (18) and (20) to derive upper limits to the coupling $C_{U} f_{A e} / m_{U}^{2}$ appearing in the Lagrangian of Eq. (14).

Figure 9 shows the upper limit, assuming an NFW halodensity profile, to the coupling $C_{U} f_{A e} / m_{U}^{2}$ from measurements of the $511-\mathrm{keV}$ line [8], the limit to the bremsstrahlung-photon flux [24], and our $2 \sigma$ limit to the

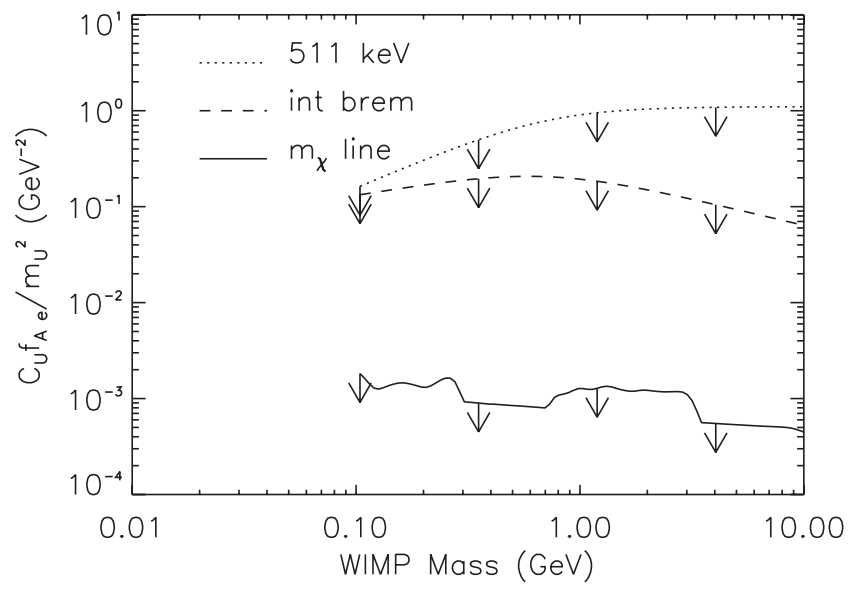

FIG. 9. Upper limits to the ratio $C_{U} f_{A e} / m_{U}^{2}$ as a function of WIMP mass for the NFW halo-density profile. The limits were calculated from the observed $511 \mathrm{keV}$ emission, the constraints on internal bremsstrahlung, and our derived limit to the linephoton flux. 
line-photon flux. We see that, for the model assumptions and WIMP mass range considered here, the limit to the two-photon annihilation cross section derived from our $2 \sigma$ limit to the line-photon flux is the strongest of these three. At first, this result may seem surprising, given that the twophoton annihilation process is higher order in $\alpha_{e}$, but this suppression is counteracted by the helicity suppression of the cross section for annihilation of Majorana fermions to electron-positron pairs. References $[28,29]$ considered also gamma rays from in-flight annihilation from $e^{+} e^{-}$pairs, but their analysis was restricted to energies $<100 \mathrm{MeV}$.

Of course, the $2 \sigma$ limit to the line-photon flux may not always provide the best limit to the two-photon annihilation cross section for every WIMP model. It may well be that other models-e.g., those in which the dark-matter particle is a scalar [30] - can produce a ratio of 511-keV photons to line photons large enough to cause the 511-keV limit to supersede the line-photon limit.

\section{ACKNOWLEDGMENTS}

We thank S. Profumo for useful discussions and comments and J. Beacom for useful comments on an earlier draft. A. R. P. was supported by the NSF. R.C. was partially funded under NASA Contract No. 1407. M. K. was supported by DOE DE-FG03-92-ER40701, NASA NNG05GF69G, and the Gordon and Betty Moore Foundation.
[1] L. Bergstrom, Rep. Prog. Phys. 63, 793 (2000).

[2] G. Bertone, D. Hooper, and J. Silk, Phys. Rep. 405, 279 (2005).

[3] G. Jungman, M. Kamionkowski, and K. Griest, Phys. Rep. 267, 195 (1996).

[4] H.E. Haber and G. L. Kane, Phys. Rep. 117, 75 (1985).

[5] A. Bottino, F. Donato, N. Fornengo, and S. Scopel, Phys. Rev. D 70, 015005 (2004).

[6] J. F. Gunion, D. Hooper, and B. McElrath, Phys. Rev. D 73, 015011 (2006).

[7] F. Ferrer, L. M. Krauss, and S. Profumo, Phys. Rev. D 74, 115007 (2006).

[8] C. Boehm, D. Hooper, J. Silk, M. Casse, and J. Paul, Phys. Rev. Lett. 92, 101301 (2004).

[9] P. Jean et al., Astron. Astrophys. 407, L55 (2003).

[10] J. Knodlseder et al., Astron. Astrophys. 411, L457 (2003).

[11] L. Bergstrom and H. Snellman, Phys. Rev. D 37, 3737 (1988).

[12] J. F. Navarro, C. S. Frenk, and S. D. M. White, Astrophys. J. 462, 563 (1996).

[13] D. J. Thompson et al., Astrophys. J. Suppl. Ser. 86, 629 (1993).

[14] R. C. Hartman et al. (EGRET Collaboration), Astrophys. J. Suppl. Ser. 123, 79 (1999).

[15] S. D. Hunter et al., Astrophys. J. 481, 205 (1997).
[16] T. D. Willis, arXiv:astro-ph/0201515.

[17] D. L. Bertsch, T. M. Dame, C. E. Fichtel, S. D. Hunter, P. Sreekumar, J.G. Stacy, and P. Thaddeus, Astrophys. J. 416, 587 (1993).

[18] J. M. Fierro, P.F. Michelson, P. L. Nolan, and D. J. Thompson, Astrophys. J. 494, 734 (1998).

[19] P. Sreekumar, F. W. Stecker, and S.C. Kappadath, AIP Conf. Proc. 510, 459 (2000).

[20] A.V. Kravtsov, A. A. Klypin, J.S. Bullock, and J.R. Primack, Astrophys. J. 502, 48 (1998).

[21] L. Bergstrom, P. Ullio, and J. H. Buckley, Astropart. Phys. 9, 137 (1998).

[22] C. Boehm and P. Fayet, Nucl. Phys. B683, 219 (2004).

[23] P. Fayet, Phys. Rev. D 70, 023514 (2004).

[24] J. F. Beacom, N. F. Bell, and G. Bertone, Phys. Rev. Lett. 94, 171301 (2005).

[25] H. Goldberg, Phys. Rev. Lett. 50, 1419 (1983).

[26] R. L. Kinzer et al., Astrophys. J. 559, 282 (2001).

[27] S. Rudaz, Phys. Rev. D 39, 3549 (1989).

[28] J. F. Beacom and H. Yuksel, Phys. Rev. Lett. 97, 071102 (2006).

[29] P. Sizun, M. Casse, and S. Schanne, Phys. Rev. D 74, 063514 (2006).

[30] C. Boehm, J. Orloff, and P. Salati, Phys. Lett. B 641, 247 (2006). 